P1: GRA

The Journal of Risk and Uncertainty $\quad$ SJNW524-03-NO00005104 $\quad$ October 24, $2005 \quad$ 19:23

The Journal of Risk and Uncertainty, 31:3; 289-300, 2005

\title{
1 Fear of Ruin
}

2 JÉRÔME FONCEL

3 Gremars, University of Lille 3, France

4 NICOLAS TREICH*

ntreich@toulouse.inra.fr

5 Lerna-Inra, University of Toulouse, France

\section{Abstract}

7 This paper offers interpretations and applications of the "fear of ruin" coefficient (Aumann and Kurz, 1977,

8 Econometrica). This coefficient is useful for analyzing the behavior of expected utility maximizers when they face

9 binary lotteries with the same worse outcome. Comparative statics results of "more fear of ruin" are derived. The

10 partial ordering induced by the fear of ruin coefficient is shown to be weaker than that induced by the Arrow-Pratt

11 coefficient.

12 Keywords: risk-aversion, expected utility, arrow-Pratt coefficient, auctions, value-of-life

13 JEL Classification: D81

14 This paper offers various interpretations of a risk-aversion coefficient, $[u(w)-u(0)] / u^{\prime}(w)$ in 15 standard notation, that was first introduced by Aumann and Kurz (1977). This coefficient, 16 coined the "fear of ruin" $(F R)$ coefficient, captures an individual's "attitude toward risking 17 his fortune." "There has not been to date any systematic analysis of this coefficient. This 18 paper fills this gap by identifying situations in which the $F R$ coefficient controls the behavior 19 of expected utility maximizers. These situations involve choices among binary lotteries with 20 a fixed worse outcome.

\section{1. Fear of ruin in the small and in the large}

22 How much would an individual be willing to pay to be fully insured against the possibility 23 of ruin? Suppose that this individual maximizes his expected utility, with an increasing von 24 Neumann Morgenstern utility function $u$ and current wealth $w$ (assume $w>0$ ). He may 25 lose his entire wealth $w$ with probability $p$. The insurance premium $z(p)$ is defined by ${ }^{2}$

$$
u(w-z(p))=(1-p) u(w)+p u(0)
$$

\footnotetext{
*To whom correspondence should be addressed.

${ }^{1}$ Aumann and Kurz note that this interpretation is an outcome of a conversation they had with Kenneth Arrow.

${ }^{2}$ The model could allow any arbitrary wealth $\underline{w}$ in the case of ruin. Here, we simply assume, without loss of generality, that $\underline{w}=0$. We also assume that $u(0)$ is finite.
} 
Assume that $u$ is differentiable. Differentiating (1) with respect to $p$ gives $z^{\prime}(p)=\frac{u(w)-u(0)}{u^{\prime}(w-z(p))}$. Suppose that the probability $p$ is small enough so that $z(p)$ may be reasonably approximated by $p z^{\prime}(0)$ since $z(0)=0$. A first-order Taylor approximation of the insurance premium $z(p)$ is then given by $z(p) \approx p \frac{u(w)-u(0)}{u^{\prime}(w)}$.

This insurance premium "in the small" thus depends separately on the characteristics of the risk and of the individual. In accord with intuition, the premium is proportional to the probability of ruin $p$. Moreover, it depends on the characteristics of the individual's utility function only through the ratio $\frac{u(w)-u(0)}{u^{\prime}(w)}$. Observe that this ratio is invariant to affine transformations of utility.

As in Aumann and Kurz (1977), we scale the utility function by assuming $u(0)=0$. This is done for expositional convenience and without loss of generality since the utility function is defined up to an affine transformation. In this case, the insurance premium is

$$
z(p) \approx p \frac{u(w)}{u^{\prime}(w)}
$$

The coefficient $u / u^{\prime}$ corresponds to the "fear of ruin" coefficient, as it was first introduced by Aumann and Kurz (1977). From now, and throughout the paper, we will refer to $u / u^{\prime}$ as the coefficient of fear of ruin, or $F R^{3}$

Observe that our approximation for the insurance premium does not directly depend on the Arrow-Pratt coefficient, $-u^{\prime \prime} / u^{\prime}$. This is because we approximate this premium for a small change in the probability $p$, not for a small change in the variation of terminal wealth, as in Pratt (1964) and Arrow (1971). Consequently, we can derive a first-order approximation of the insurance premium by simply examining the rate of increase of the insurance premium with respect to $p .^{4}$

Furthermore, observe that $u(w) / u^{\prime}(w)$ is always strictly positive under $u(\cdot)$ increasing, since $u(0)=0$. Moreover, under risk-aversion, it is easy to see that this coefficient always increases with wealth $w$. Intuitively, there are two reasons why the insurance premium increases with wealth. First, when the agent is wealthier, there is more to lose. As a result, the agent is willing to pay more in the face of the risk of losing his entire wealth. This is the effect related to the numerator, $u(w)$, which increases in $w$. Second, under risk-averse preferences, the marginal value of money is smaller when the agent is wealthier, so he is willing to sacrifice a larger amount of money in face of the same risk. This effect is related to the term $1 / u^{\prime}(w)$, which also increases in $w$ under risk-aversion.

Also, simply observe that, under risk neutrality, the FR coefficient reduces to $w$. Moreover, if $u$ is concave,

$$
\frac{u(w)}{w} \geq u^{\prime}(w)
$$

This inequality states that the slope of the tangent to the utility function at $w$ is always smaller than the slope of the chord drawn from 0 to $w$. Multiplying both sides of this

\footnotetext{
${ }^{3}$ Hence, the reader should remember that the appropriate $F R$ coefficient in the general case is $[u(w)-u(0)] / u^{\prime}(w)$.

${ }^{4}$ The equivalent first-order effect for a variation in terminal wealth is zero in Pratt, so that he examines the second order effect. See Gollier (2001, p. 21-24) for a detailed analysis of Pratt (1964)'s "in the small" approximation.
} 
60 inequality by $w / u^{\prime}(w)$ shows that the $F R$ coefficient is always larger under risk-aversion

61 than under risk-neutrality. This is consistent with the intuitive requirement that one's fear 62 of ruin is lower when one is risk-neutral. ${ }^{5}$

63 Previous remarks interpret $F R$ as a measure of local risk aversion or local propensity to 64 insure against a small chance of ruin. We next examine comparative properties of the $F R$ coefficient for any probability of ruin $p$. To do so, we first introduce two Definitions.

Definition 1. We define $z(u, w, p)$, the insurance premium of agent $u$ facing the risk of losing wealth $w$ with probability $p$, by

$$
u(w-z(u, w, p))=(1-p) u(w)
$$

68

Definition 2. We define $c(u, w, p)$, the compensating premium of agent $u$ facing the risk of losing wealth $w$ with probability $p$, by

$$
u(w)=(1-p) u(w+c(u, w, p))
$$

The quantity $z(u, w, p)$ is the insurance premium that agent $u$ with current wealth $w$ is willing to pay to avoid the possibility that a ruin occurs with probability $p$. The quantity $c(u, w, p)$ is the compensating premium that agent $u$ is willing to accept to face a possibility of ruin, namely to end up with wealth 0 with probability $p$ or with wealth $w+c(u, w, p)$ otherwise.

Following the approach developed by Pratt (1964), we now compare the FR of two individuals $u$ and $v$ for all $w$ and $p$. Under the normalization at 0 adopted above, we introduce the following natural definition of "more fear of ruin".

Definition 3. Agent $v$ is said to have more fear of ruin $(F R)$ than agent $u$ if and only if for all $w$,

$$
\frac{v(w)}{v^{\prime}(w)} \geq \frac{u(w)}{u^{\prime}(w)} .
$$

80 Using the three Definitions above, we can now state the first Proposition of this paper. ${ }^{6}$

81 Proposition 1. Consider two agents with strictly increasing and differentiable utility functions $u$ and $v$ such that $u(0)=v(0)=0$. For all $p \in[0,1]$ and all strictly positive wealth $w$, the following four conditions are equivalent:

84 (i) Agent $v$ has more I than agent $u$, namely $\frac{v(w)}{v^{\prime}(w)} \geq \frac{u(w)}{u^{\prime}(w)}$;

85 (ii) Agent $v$ has a higher insurance premium than agent $u$, namely $z(v, w, p) \geq z(u, w, p)$;

86 (iii) Agent $v$ has a higher compensating premium than $\bar{u}$, namely $c(v, w, p) \geq c(u, w, p)$;

\footnotetext{
${ }^{5}$ Moreover observe that the fear of ruin is even lower when preferences are risk-seeking.

${ }^{6}$ Detailed proofs are available upon request. See also Foncel and Treich (2003).
} 
(iv) There exists an increasing and differentiable function $T(\cdot)=v \circ u^{-1}(\cdot)$ such that $T(0)=0$ and for all $x, \frac{T(x)}{x}$ is decreasing in $x$.

A sketch of the proof follows. We prove the equivalence between (i), (ii) and (iv). First, (ii) implies (i) by (2). Second, we show that (i) implies (iv). Observe that since $u$ and $v$ are increasing and differentiable functions, there always exists a unique, increasing and differentiable function $T=v \circ u^{-1}$ such that $v=T \circ u$. Also, $v(0)=T \circ u(0)=T(0)=0$. Moreover, from $\frac{u}{u^{\prime}} \leq \frac{v}{v^{\prime}}$, we have $T^{\prime}(u) \leq \frac{T(u)}{u}$, which must be true for all $u$. This latter condition is equivalent to (iv). Third, we show that (iv) implies (ii). By Definition 1

$$
v(w-z(u, w, p))=T(u((w-z(u, w, p)))=T((1-p) u(w))
$$

Since $T(x) / x$ is decreasing in $x$, we get $T((1-p) u(w)) \geq(1-p) T(u(w))$. We thus have $v(w-z(u, w, p)) \geq(1-p) v(w)=v(w-z(v, w, p))$. This implies (ii). The proof of the equivalence between (i), (iii) and (iv) is similar.

The result would trivially generalize to a risk premium $\pi(u, w, p)$, namely the insurance premium net of the expected value of the risk $\pi(u, w, p)=z(u, w, p)-p w$.

\section{Applications}

In this section, we show that the $F R$ coefficient is applicable to a wide variety of models. $\mathbf{1 0 2}$ Consistent with the previous section, these applications involve choices among lotteries $\mathbf{1 0 3}$ with just two possible outcomes in which the worse outcome of the lotteries is the same, 104 equal to the "ruin point" (normalized to zero).

\subsection{Value-of-statistical-life}

Let us interpret $u(0)$ in model (1) as the utility when dead. In other words, the ruin point is $\mathbf{1 0 7}$ the death point. The expected utility equals $(1-p) u(w)$; there is no bequest motive. The $\mathbf{1 0 8}$ value-of-statistical-life (VSL) is usually defined as the rate of substitution between wealth $\mathbf{1 0 9}$ $w$ and mortality risk $p$ (see, e.g., Viscusi, 1993). We have

$$
\mathrm{VSL}=\frac{d w}{d p}=\frac{u(w)}{(1-p) u^{\prime}(w)}=\frac{F R[u(w)]}{(1-p)}
$$

where $F R[u(\cdot)] \equiv u(\cdot) / u^{\prime}(\cdot)$. In this simple model, it is clear that there is a one-to-one 111 relation between VSL and $F R$. An individual has, other things being equal, a higher VSL if 112 and only if he has more $F R$.

Let us slightly adapt the model now to allow for insurance opportunities. More precisely, 114 assume that there is an annuity market in which survivors are offered fair tontines shares $\mathbf{1 1 5}$ (Rosen, 1988). In a large group of identical individuals, a proportion $p$ die and their wealth $\mathbf{1 1 6}$ is distributed to $(1-p)$ survivors. A survivor's consumption thus equals initial wealth $w \mathbf{1 1 7}$ 
118 plus the tontine share $p w /(1-p)$, that is a total of $w /(1-p)$. The state-dependent expected 119 utility thus equals $(1-p) u(w /(1-p))$ and we have

$$
\mathrm{VSL}=F R[u(w /(1-p))]-\frac{w}{1-p}
$$

120 There is still a one-to-one relation between VSL and FR in this model introduced by Rosen 121 (1988).

122 Interestingly, $F R$ plays an important role in a life-cycle model as well. To see this point, 123 consider the following two-period model

$$
V \equiv \max _{c} u(c)+\beta(1-p) u(R(w-c))
$$

124

${ }^{7}$ Garber and Phelps (1997) indicate that $u / u^{\prime}$ is a "central component" in their lifetime medical spending model. Also, in a recent unpublished paper, Bommier (2003) shows that $F R$ is a crucial coefficient when one wants to compare lotteries involving lives of different lengths. He calls the $F R$ coefficient the "general rate of substitution between the length of life and consumption at the end of life".

${ }^{8}$ See for instance Milgrom (2004, pages 123-125). 
What is the effect of increased risk aversion in the sense of more $F R$ on the equilibrium bidding function $B(x)$ ? Assume that bidders $v$ have more $F R$ and let us compare, ceteris paribus, the outcome of a first-price auction populated by bidders $v$ instead of bidders $u .^{9}$ Using straightforward notation we find

$$
\begin{aligned}
B_{v}^{\prime}(x)-B_{u}^{\prime}(x) & =(N-1) \frac{f(x)}{F(x)}\left[\frac{v\left(x-B_{v}(x)\right)}{v^{\prime}\left(x-B_{v}(x)\right)}-\frac{u\left(x-B_{u}(x)\right)}{u^{\prime}\left(x-B_{u}(x)\right)}\right] \\
& \geq(N-1) \frac{f(x)}{F(x)}\left[\frac{u\left(x-B_{v}(x)\right)}{u^{\prime}\left(x-B_{v}(x)\right)}-\frac{u\left(x-B_{u}(x)\right)}{u^{\prime}\left(x-B_{u}(x)\right)}\right],
\end{aligned}
$$

by $\frac{v(\cdot)}{v^{\prime}(\cdot)} \geq \frac{u(\cdot)}{u^{\prime}(\cdot)}$. From that last result, we easily find that, for all $x$,

$$
B_{v}(x)=B_{u}(x) \operatorname{implies} B_{v}^{\prime}(x) \geq B_{u}^{\prime}(x) .
$$

We thus have obtained a single crossing property. This property means that $B_{v}$ can only $\mathbf{1 4 2}$ cross $B_{u}$ from below. Since $B_{v}(\underline{x})=B_{u}(\underline{x})=\underline{x}$, the function $B_{v}(x)$ will always be larger $\mathbf{1 4 3}$ than $B_{u}(x)$ for any $x$ such that $x \geq \underline{x}$. Therefore, more $F R$ always raises the bidding price 144 equilibrium. This finding leads to the following Proposition.

Proposition 2. The equilibrium price of a first-price auction with independent private $\mathbf{1 4 6}$ values increases when bidders have more FR.

This result extends that of Milgrom and Weber (1982), who showed that introducing 148 risk-aversion raises the bidding price compared to the risk-neutral case.

\subsection{Conflict and bargaining games}

A conflict game may be described as follows (see, e.g., Skaperdas, 1997). Two agents, say 1151 and 2, possess one unit of a resource. They may convert this resource and invest it into arms, 152 in quantities $y_{1}$ and $y_{2}$ respectively. The winner of the conflict gets a prize that depends on $\mathbf{1 5 3}$ the remaining productive resources of both agents, while the loser gets 0 . The prize is a $\mathbf{1 5 4}$ function $C \equiv C\left(1-y_{1}, 1-y_{2}\right)$ which is increasing in both arguments. Let $p \equiv p\left(y_{1}, y_{2}\right) \mathbf{1 5 5}$ and $1-p$ denote the winning probability of agent 1 and 2 respectively, and $u_{1}$ and $u_{2}$ their $\mathbf{1 5 6}$ utilities so that they respectively maximize

$$
p u_{1}(C) \text { and }(1-p) u_{2}(C) .
$$

It can be shown that, in such a game, an agent with more $F R$ always invests more into arms $\mathbf{1 5 8}$ and has a higher probability of winning the conflict when $C$ is symmetrical. See Skaperdas $\mathbf{1 5 9}$ (1997, page 117, equation. 4). Moreover, when two identical agents simultaneously have $\mathbf{1 6 0}$

\footnotetext{
${ }^{9}$ The assumption that private values are independent of private characteristics, like risk-aversion, obviously facilitates the comparative statics analysis here.
} 
161 162

more $F R$, the total amount invested into arms increases as well. The intuition for this is straightforward. On the one hand, increasing investment into arms decreases payoff $C$ in the case of victory. On the other hand, increasing investment decreases the chance of losing the conflict, and so helps to avoid ruin (notice that the loser's payoff is the ruin point here). The trade-off is thus similar to the one presented in the previous models. It is not surprising that FR controls the amount of resources invested into arms in this model. ${ }^{10}$

Another application in strategic games is the Nash bargaining problem, as first noticed by Aumann and Kurz (1977, p. 1149). To see that, consider two agents $u_{1}$ and $u_{2}$ who bargain over the division of a cake of size $w$. The well-known Nash solution to this problem calls for maximizing

$$
u_{1}\left(y_{1}\right) u_{2}\left(y_{2}\right) \quad \text { subject to } y_{1}+y_{2}=w
$$

It is easy to show that this solution equates the two individuals' FR computed at the optimal bargaining points. The intuition is as follows (see also Svejnar, 1986): In the bargaining problem, the ruin point $u(0)=0$ can be interpreted as the threat utility if the bargaining process fails. As a result, at each stage of the bargaining process, each agent considers a gamble in which he risks losing the entire net gain which he has won so far against an additional gain of a small amount. More fear of ruin thus reduces the willingness to accept this gamble and so is a disadvantage in bargaining. See Roth and Rothblum (1982) for a general analysis.

\subsection{Contingent background risk}

Take model (1) but replace the term $u(w)$ by the term $u_{\varepsilon}(w) \equiv E_{\varepsilon} u(w+\tilde{\varepsilon})$ and assume $E \tilde{\varepsilon}=0$. The individual thus faces a background risk $\tilde{\varepsilon}$ only if ruin does not occur. What is the effect of this contingent background risk? From Proposition 1, it is clear that the insurance premium always decreases if and only if

$$
\frac{u_{\varepsilon}}{u_{\varepsilon}^{\prime}} \leq \frac{u}{u^{\prime}}
$$

Observe that, given risk-averse preferences, $E \tilde{\varepsilon}=0$ implies $u_{\varepsilon}(\cdot)=E u(.+\tilde{\varepsilon}) \leq u(\cdot)$ by the Jensen inequality. Similarly, given prudence, $E \tilde{\varepsilon}=0$ implies $u_{\varepsilon}^{\prime}(\cdot)=E u^{\prime}(.+\tilde{\varepsilon}) \geq u^{\prime}(\cdot)$ by the Jensen inequality. Hence, under the conditions of positive risk-aversion and prudence, FR decreases with a contingent background risk. Some implications directly follow. For instance, the VSL of risk-averse and prudent individuals decreases in face of a background risk contingent on being alive. ${ }^{11}$

\footnotetext{
${ }^{10}$ The $F R$ coefficient is also at play in contest games (Skaperdas and Gan, 1995), or in rent-augmenting and rent-seeking games (Konrad and Schlesinger, 1997). However, more $F R$ is not enough to control the comparative statics of more risk-aversion in those games as the loser's payoff generally depends on the agents' actions, and so the ruin point varies.

${ }^{11}$ This observation relates to Eeckhoudt and Hammitt (2001)'s analysis of the effect of a financial background risk on the VSL.
} 
Let us now consider an implication of this observation concerning the first-price auction 190 model. This implication arises when the value of the auctioned object is uncertain. Here, 191 we follow (Eso and White, 2004). Take the standard model of Section 3.2. Assume that the 192 private value of the auctioned object is no longer $x_{i}$ but instead is $x_{i}+\varepsilon_{i}$, where $\varepsilon_{i}$ is the 193 realization of a random variable $\tilde{\varepsilon}_{i}$. Random variables $\tilde{\varepsilon}_{i}$ are identically distributed as $\tilde{\varepsilon}, 194$ and are independent of private values $x_{i}$. Thus, the highest bidder now receives an ex post 195 payoff $x_{i}+\varepsilon_{i}-b_{i}$. Losing bidders still receive payoff of 0 . This model implies that the $\mathbf{1 9 6}$ background risk is contingent upon winning the auction. Ex ante, agent $i$ chooses $b_{i}$ so as 197 to maximize

$$
p_{i} E_{\varepsilon} u\left(x_{i}+\tilde{\varepsilon}-b_{i}\right) \equiv p_{i} u_{\varepsilon}\left(x_{i}-b_{i}\right)
$$

with $p_{i}$ defined as above. It is immediately clear that the differential equation characterizing 199 the equilibrium strategy in the noisy auction takes on the following form

$$
B^{\prime}(x)=(N-1) \frac{f(x)}{F(x)} \frac{u_{\varepsilon}(x-B(x))}{u_{\varepsilon}^{\prime}(x-B(x))}, \quad \text { with } B(\underline{x})=\underline{x} .
$$

In other words, analyzing the effect of the noise $\tilde{\varepsilon}$ on the equilibrium bidding price amounts 201 to comparing the equilibrium with utilities $u_{\varepsilon}(\cdot)$ to the equilibrium with utilities $u(\cdot)$. This $\mathbf{2 0 2}$ leads to the following Proposition.

Proposition 3. Consider a first-price auction with independent private values and with 204 risk-averse and prudent bidders. Then uncertainty over the value of the auctioned object $\mathbf{2 0 5}$ decreases the equilibrium price.

The intuition is two-fold. First, when preferences are risk-averse, utility is reduced if one $\mathbf{2 0 7}$ wins the object $u_{\varepsilon}(\cdot) \leq u(\cdot)$. Hence, the object is less desirable. Second, given prudence, the $\mathbf{2 0 8}$ marginal utility of income increases $u_{\varepsilon}^{\prime}(\cdot) \geq u^{\prime}(\cdot)$. Individuals thus bid less aggressively in 209 the noisy auction because they value an extra dollar of income more. This Proposition shows 210 that Eso and White (2004)'s result that decreasing absolute risk-averse (DARA) individuals 211 bid smaller amounts in a noisy first-price auction also holds for any risk-averse prudent $\mathbf{2 1 2}$ bidders (i.e., DARA is sufficient for prudence but the converse is not true).

Overall, these applications suggest that $F R$ may be useful to sign various comparative $\mathbf{2 1 4}$ statics results in a large class of models used throughout the economics literature.

\section{Comparison with the Arrow-Pratt and the asymptotic risk-aversion coefficients 216}

We have demonstrated in the previous section that more risk-aversion in the sense of $F R \quad \mathbf{2 1 7}$ increases the bidding price in a model of first-price auctions, and also controls risk-aversion $\mathbf{2 1 8}$ motives in other models. This raises the question of the effect of an increase in risk-aversion $\mathbf{2 1 9}$ à la Arrow-Pratt in those models. The answer to the question is given in the present section, 220 as we precisely examine the link between $F R$ and the Arrow-Pratt coefficient. 
222

Following Jones-Lee (1980), it is useful to distinguish three different risk-aversion coefficients

$$
\begin{aligned}
& F R[u(\cdot)] \equiv \frac{u(\cdot)}{u^{\prime}(\cdot)} \\
& A P[u(\cdot)] \equiv \frac{-u^{\prime \prime}(\cdot)}{u^{\prime}(\cdot)} \\
& A S[u(\cdot)] \equiv \frac{u^{\prime}(\cdot)}{u^{*}-u(\cdot)} .
\end{aligned}
$$

The last coefficient corresponds the asymptotic risk aversion coefficient (AS) introduced by Jones-Lee (1980). The AS coefficient measures the individual's willingness to participate in a "small-stake large-prize gamble." It assumes that $u$ is bounded above, where $u$ * is the supremum of $u .^{12}$

The complementarity of these three coefficients is apparent when one approximates insurance premia "in the small." Indeed, it is well-known that the Arrow-Pratt coefficient appears when considering risks with small gains and small losses. On the other hand, we have seen that the FR coefficient appears when the risk is a small probability of ruin. Finally, the AS risk aversion coefficient appears for a small loss/large gain risk, like gambling for the jackpot. See Jones-Lee (1980) for an interesting presentation and discussion.

In this section, we ask: to what extent is an individual $v$ who is more risk-averse than an individual $u$ in one specific sense also more risk-averse with respect to another sense? In other words, we want to compare the partial orderings induced by these three risk-aversion coefficients. To do so, let the statement " $v$ is more risk averse than $u$ in the sense of $I$ " be condensed into $v \supseteq_{I} u$ and defined as follows.

Definition 4. Consider the three coefficients $I=\{F R, A P, A S\}$ as they are introduced in (3). Then

(i) $v \supseteq_{F R} u$ holds if and only if $F R[v(w)] \geq F R[u(w)]$ for all $w$,

(ii) $v \supseteq_{A P} u$ holds if and only if $A P[v(w)] \geq A P[u(w)]$ for all $w$,

(iii) $v \supseteq_{A S} u$ holds if and only if $A S[v(w)] \geq A S[u(w)]$ for all $w$.

Conversely, the ordering $v \quad \nsupseteq \quad{ }_{I} u$ means that there exists $w$ such that $u$ is locally more risk averse than $v$ in the sense of $I$.

The claim that an individual $v$ is more risk averse than an individual $u$ (in the sense of $A S$, $A P$ or $F R$ ) can be fully characterized by setting the corresponding properties on a function $T$ such that $v=T \circ u$. First, from Pratt (1964, Theorem 1) we know that $v \supseteq_{A P} u$ if and only if $v=T \circ u$ with $T$ increasing, twice differentiable and concave. Second, from Proposition 1, we know that $v \supseteq_{F R} u$ if and only if $v=T \circ u$ where $T$ is increasing, differentiable, $T(0)=0$ and $T(x) / x$ decreasing in $x$. Finally, it is easy to show that $v \supseteq_{A S} u$ if and

\footnotetext{
${ }^{12}$ What is actually important is that both the ruin point and $u^{*}$ are very bad and very good points beyond which it is not possible to go. In particular, the utility $u$ needs not be bounded above if we set an upper limit for wealth.
} 
P1: GRA

The Journal of Risk and Uncertainty $\quad$ SJNW524-03-NO00005104 October 24, $2005 \quad$ 19:23

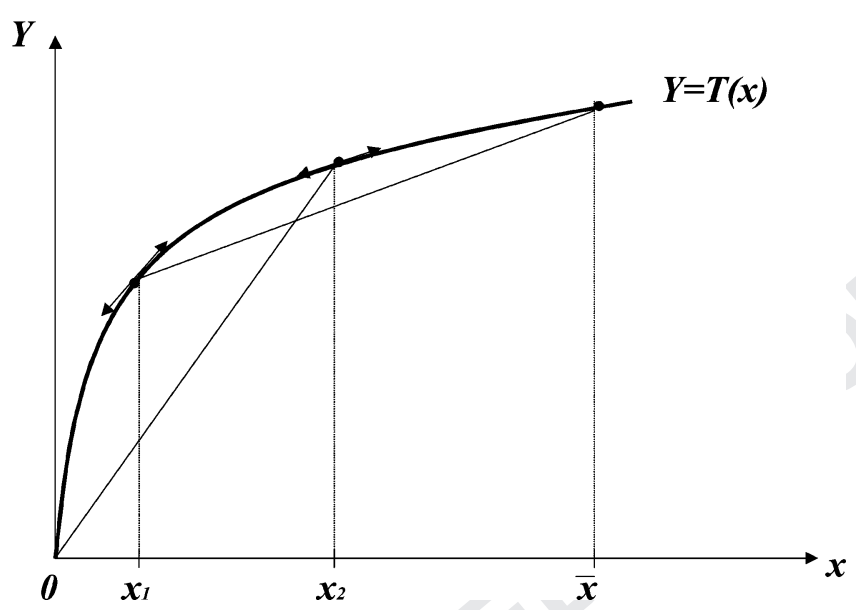

Figure 1. Represents an increasing and concave function $T$ with $T(0)=0$. This function is such that $T(x) / x>$ $T^{\prime}(x)$ together with $T^{\prime}(x)>\frac{T(\bar{x})-T(x)}{\bar{x}-x}$ for all $x \in[0, \bar{x}]$.

only if $v=T \circ u$ where $T$ is increasing, differentiable on $[0, \bar{x}]$ and $T^{\prime}(x) \geq \frac{T(\bar{x})-T(x)}{\bar{x}-x}, 252$ with $\bar{x}=u^{*}=\sup _{w} u(w)$. Hence, the comparative analysis of the different risk-aversion 253 coefficients can be presented by equivalent characterizations on such transformations $T \quad \mathbf{2 5 4}$ without any reference to the underlying utility functions $u$ and $v{ }^{13}$

It is immediate that if a function $T$ is concave on $[0, \bar{x}]$ then $T(x) / x$ is decreasing in $x, 256$ which is equivalent to $T(x) / x>T^{\prime}(x)$, and to $T^{\prime}(x) \geq \frac{T(\bar{x})-T(x)}{\bar{x}-x}$. Figure 1 illustrates this 257 result. First, observe on the figure that the slope of the chord drawn from the end-point $\bar{x} \mathbf{2 5 8}$ to any point $x_{1}$, i.e. $\frac{T(\bar{x})-T\left(x_{1}\right)}{\bar{x}-x_{1}}$, is always lower than the slope of the tangent at this point 259 $T^{\prime}\left(x_{1}\right)$. Second, observe that the slope of the chord drawn from the origin to any point $x_{2}, \mathbf{2 6 0}$ i.e. $T\left(x_{2}\right) / x_{2}$, is larger than the slope of the tangent at this point $T^{\prime}\left(x_{2}\right)$. The results for $\mathbf{2 6 1}$ partial orderings are summarized as follows.

Proposition 4. Let $u$ and $v$ be two strictly increasing, twice differentiable and concave $\mathbf{2 6 3}$ functions that are bounded above with $u^{*}=\sup _{w} u(w)$ and $v^{*}=\sup _{w} v(w)$. Moreover, 264 assume that $u(0)=v(0)=0$. Then $v \supseteq_{A P} u$ implies:

(ii) $v \supseteq_{A S} u$.

This Proposition shows that if an agent is more risk-averse in the classical sense of $A P$ then $\mathbf{2 6 8}$ he is also more risk-averse in the sense of $A S$ and $F R$. Proposition 4 is of clear mathematical 269 significance. As mentioned above, $v$ is more risk-averse than $u$ in the sense of $A P$ if and $\mathbf{2 7 0}$ only if $v$ is obtained by a concave transformation of $u$. This is a very intuitive mathematical 271 property, as any coefficient of curvature of a function should in principle increase when one 272

\footnotetext{
${ }^{13}$ In order to compare these different characterizations $T$, we need to restrict our attention to any increasing, differentiable $T$ defined over $[0, \bar{x}]$ and such that $T(0)=0$.
} 
273

"concavifies" a function. The Proposition shows that this is actually the case for $A S$ and $F R$ coefficients.

Proposition 4 shows that $\supseteq_{F R}$ and $\supseteq_{A S}$ are weaker orderings than $\supseteq_{A P}$. Moreover, it is possible to show that these orderings are strictly weaker. To see this, take the function $T_{1}(x)=$ $(x-2)^{3}+8$ over the interval $[0, \bar{x}]$ where $\bar{x}=2.8$. This function is such that $T_{1}(x) / x$ is decreasing in $x$ over this entire interval while $T_{1}^{\prime \prime}(x)>0$ together with $\frac{T_{1}(\bar{x})-T_{1}(x)}{\bar{x}-x}>T_{1}^{\prime}(x)$ for some $x$ over this interval. In other words, there exists an individual $v$ who has globally more $F R$ than $u$ but who is also locally less risk-averse than $u$ in the sense of $A S$ and of $A P$. Similarly, let $T_{2}(x)=\bar{x}-2-\left[T_{1}(\bar{x})-x-8\right]^{1 / 3}$ over the interval $\left[0, T_{1}(\bar{x})\right]$ where $T_{1}$ is the function just defined above. ${ }^{14}$ Function $T_{2}$ is such that $\frac{T_{2}\left(T_{1}(\bar{x})\right)-T_{2}(x)}{T_{1}(\bar{x})-x}<T_{2}^{\prime}(x)$ over this entire interval while $T_{2}(x) / x$ is increasing in $x$ and $T_{2}^{\prime \prime}(x)>0$ for some $x$ over this latter interval. In other words, there also exists an individual $v$ who is globally more risk-averse in the sense of $A S$ than $u$ but who is locally less risk-averse in the sense of $F R$ and of $A P$.

To conclude this section, we remark that $\supseteq_{F R}, \supseteq_{A S}$ and $\supseteq_{A P}$ are equivalent for some important classes of utility functions. This is the case if we restrict our attention to power functional forms. Technically, the curvature of power functions is often captured by one single parameter and the $A P, F R$ and $A S$ coefficients may vary monotonically with this parameter. ${ }^{15}$ The equivalence result follows.

\section{Conclusion}

In this paper, we have investigated the basic properties of the "fear of ruin" $(F R)$ coefficient introduced by Aumann and Kurz (1977). First, we have derived an approximation of the insurance premium that an individual would be willing to pay in face of a small chance of losing his entire wealth. This premium has been shown to be proportional to the $F R$ coefficient. We have then provided equivalent characterizations for comparing the $F R$ of two agents. Specifically, we have shown that an agent $v$ has globally more $F R$ than an agent $u$ if and only if $v$ 's premium to insure against the risk of ruin is always larger than $u$ 's premium. We also have given a characterization of more $F R$ in terms of the properties required of an increasing transformation $T$, such that $v=T \circ u$. Furthermore, we have shown that the FR coefficient plays a crucial role in strategic games with risk-averse players. For instance, in first price auctions, we have demonstrated that the equilibrium bidding price of an auctioned object is always higher if auctioneers have more FR, and that uncertainty over the value of the auctioned object always leads the equilibrium bidding price to decrease under prudence. In addition, we have shown that the $F R$ coefficient may be instrumental in simple mortality risk models. Finally, we have compared the FR's coefficient with other

\footnotetext{
${ }^{14}$ Observe that $T_{1}$ appears in the characterization of $T_{2}$. This can be easily understood once we explain how these counter-examples were generated. In short, we used the fact that finding $T_{1}$ such that we have $\supseteq_{F R}$ and $\supseteq_{A S}$ is equivalent, up to a change of reference axes, to finding $T_{2}$ such that $\supseteq_{F R}$ and $\supseteq_{A S}$. Mathematically, the change of reference axes is such that $T_{2}(x)=\bar{x}-T_{1}^{-1}\left(T_{1}(\bar{x})-x\right)$.

${ }^{15}$ Foncel and Treich (2003) derive a formal proof of this equivalence for a generic class of power utility functions. This generic class $U_{z}$ is the class of increasing and concave function of $w$ that takes the form $\frac{(z+w)^{1-m}}{1-m}-\frac{z^{1-m}}{1-m}$, and defined for all positive parameter $m \neq 1$ and over the interval $[0, \bar{w}]$. (This result does not hold for all functions with a single parameter of power form.)
} 
P1: GRA

The Journal of Risk and Uncertainty $\quad$ SJNW524-03-NO00005104 October 24, $2005 \quad 19: 23$

coefficients of risk-aversion. In particular, we have shown that if an agent $v$ is more risk- $\mathbf{3 0 7}$ averse than $u$ in the sense of Arrow-Pratt, then $v$ has more $F R$ than $u$.

We thank Monique Chyba, Louis Eeckhoudt, Kevin Haninger, Frédéric Jouneau, François 310 Salanié and the Editor Kip Viscusi together with EGRIE participants at Zurich in 2003 for 311 their comments. We also thank a referee for his extremely helpful remarks.

\section{References}

Arrow, Kenneth J. (1971). "The Theory of Risk Aversion,” In Essays in the Theory of Risk-Bearing, Chapter 3, 314 Markham Publishing Company.

Aumann, Robert J. and Mordecai Kurz. (1977). "Power and Taxes," Econometrica 45, 1137-1161. 316

Bommier, Antoine. (2003). "Valuing Life Under the Shadow of Death," mimeo, University of Toulouse. $\quad 317$

Eeckhoudt, Louis and James K. Hammitt. (2001). "Background Risks and the Value of a Statistical Life," Journal 318

of Risk and Uncertainty 23, 261-279.

Eso, Peter and Lucy White. (2004). "Precautionary Bidding in Auctions," Econometrica 72, 77-92. 320

Foncel, Jérome and Nicolas Treich. (2003). "Fear of Ruin," mimeo, Lerna, University of Toulouse, at 321 http://w3.toulouse.inra.fr/leerna/english/publicationdeng.htm.

Garber, Alan M. and Charles E. Phelps. (1997). "Economic Foundations of Cost-Effectiveness Analysis," Journal 323

of Health Economics 16, 1-31.

Gollier, Christian. (2001). The Economics of Risk and Time. Cambridge: MIT Press. 335

Jones-Lee, Michael W. (1980) "Maximum Acceptable Physical Risk and a New Measure of Financial Risk 326

Aversion," The Economic Journal 90, 550-568.

Kimball, Miles S. (1990). "Precautionary Savings in the Small and in the Large," Econometrica 58, 53-73. 328

Konrad, Kai A. and Harris Schlesinger. (1997). "Risk-Aversion in Rent-Seeking and Rent-Augmenting Games," 329

The Economic Journal 107, 1671-1683.

Milgrom, Paul R. (2004). Putting Auction Theory to Work. Cambridge University Press.

Milgrom, Paul R. and R. J. Weber. (1982). "A Theory of Auctions and Competitive Biddings," Econometrica 50, 332 $1189-1122$.

Pratt, John W. (1964). "Risk Aversion in the Small and in the Large," Econometrica 32, 122-136.

Rosen, Sherwin. (1988). "The Value of Changes in Life Expectancy," Journal of Risk and Uncertainty 1, 285-304, 335

Roth, Alvin E. and Uriel G. Rothblum. (1982). "Risk Aversion and Nash's Solution for Bargaining Games with 337

Risky Outcomes," Econometrica 50, 639-647.

Skaperdas, Stergios. (1992). "Conflicts and Attitudes Toward Risk," American Economic Review Papers and 339

Proceedings 81, 116-120.

Skaperdas, Stergios and Li Gan. (1995). "Risk-Aversion in Contests," The Economic Journal 105, 951-962. 341

Svejnar, Jan. (1986). "Bargaining Power, Fear of Disagreement and Wage Settlements: Theory and Evidence from 342

U.S. Industry," Econometrica 54, 1055-1078.

Viscusi, W. Kip. (1993). “The Value of Risks to Life and Health,” Journal of Economic Literature 31, 1912-1946. 344 